\title{
Platform Presentations
}

\section{CACN Chair's Select Abstracts}

\begin{abstract}
A.01
Targeted analysis of whole exome sequencing and genotypephenotype correlation in epileptic encephalopathies

SE Buerki (Vancouver) EB Toyota (Vancouver) I Guella (Vancouver) M McKenzie (Vancouver) D Evans (Vancouver) S Adam (Vancouver) MI van Allen (Vancouver) $C$ Boelman (Vancouver) $G$ Horvath (Vancouver) CD Van Karnebeek (Vancouver) P Eydoux (Vancouver) L Huh (Vancouver) A Datta (Vancouver) KA Selby (Vancouver) A Michoulas (Vancouver) TN Nelson (Vancouver) MJ Farrer (Vancouver) MB Demos (Vancouver)* MB Connolly (Vancouver)*
\end{abstract}

doi: 10.1017/cjn.2016.53

Background: Epileptic encephalopathy (EE) is a severe condition in which epileptic activity itself may contribute to severe cognitive and behavioural impairments above and beyond what might be expected from the underlying pathology alone. Next generation sequencing technologies such as whole exome sequencing (WES) can detect underlying genetic causes of in EE. Methods: This report describes genotype-phenotype correlation of 29 subjects with unexplained epileptic encephalopathy, in whom WES, targeting a list of 557 epilepsy-associated genes was performed. Epilepsy phenotyping was done according to current ILAE recommendations. Results: Median age at seizure onset was 14 months (range 1-48). Electroclinical syndromes were applicable for 16/29, 8/16 had a definite/likely diagnosis. 6/8 subjects with West syndrome had variants in ALG13, STXBP1, PAFAH1B1, SLC35A2, CDKL5 and ADSL. 2 patients with Dravet syndrome had variants in SCN1A and PCDH19 respectively. 4/29 had unspecified EE and definite/likely diagnosis due to STXBP1, POLG, and KCNQ2 (2) variants. 4/29 had a possible diagnosis involving GABRB3, ARHGEF9, PCDH19 and SCN3A variants. Conclusions: The high diagnostic yield (definite/likely diagnosis in $11 / 29=38 \%$ ), involving a broad variety of epilepsy-associated genes in different electroclinical syndromes justifies the diagnostic approach of early onset EE by next generation sequencing.

\section{A.02 \\ Clinical clues for autoimmunity in the etiology of autistic regression}

H Goez (Edmonton)* O Scott (Toronto) D Shi (Edmonton) D Andriashek (Edmonton) B Clark (Edmonton)

doi: 10.1017/cjn.2016.54

Background: Autistic regression (AR) accounts for $20-40 \%$ of patients with Autism Spectrum Disorder (ASD). ${ }^{1}$ Literature demonstrates specific immune changes in AR patients, ${ }^{2}$ as well as association between AR and autoimmune thyroiditis. ${ }^{3}$ Our study explores the clinical association between AR and autoimmunity, focusing on possible precipitants and familial autoimmunity, in comparison with patients with infantile autism (IA). Methods: charts of children diagnosed with ASD in 2014 were reviewed, and patients were classified as either AR or IA based on Autism Diagnostic Interview (ADI-R) criteria.4 Information regarding pregnancy, perinatal complications, febrile illness preceding the diagnosis, and family history of autoimmune conditions was collected. Results: 206 children had IA and $33 \mathrm{had}$ AR. No difference was found in prevalence of pregnancy or perinatal complications. The incidence of febrile illness in the 6 months prior to diagnosis and the prevalence of familial autoimmunity, were significantly higher in the AR group $(\mathrm{p}<0.001)$. Diabetes type I, celiac disease, autoimmune thyroiditis, and inflammatory bowel disease were more common in families of AR patients $(p<0.05)$. Conclusions: the association between AR and preceding febrile illness, as well as familial autoimmunity, supports the notion of AR as a separate entity within ASD, possibly mediated by autoimmune changes.

\section{A.03 \\ Cerebral perfusion and its relationship to post-concussion syndrome in mild traumatic brain injury: a prospective controlled cohort study}

KM Barlow (Calgary)* LD Marcil (Calgary) D Dewey (Calgary) H Carlson (Calgary) FP MacMaster (Calgary) BL Brooks (Calgary) RM Lebel (Calgary)

doi: 10.1017/cjn.2016.55

Background: Persistent post-concussive symptoms (PCS) have been linked to increased cortical network activation and decreased cerebrovascular reactivity. Decreased cerebral perfusion could help explain PCS and may be a biomarker to track recovery. Methods: Children (ages 8 to 18 years) symptomatic with PCS at one month post-injury were studied. Children who recovered following a mTBI (asymptomatic group) and healthy children acted as controls. Pseudocontinuous arterial spin labeling MRI was used to quantify cerebral blood flow (CBF). All subjects were imaged at approximately 40 days post-injury. Symptomatic group underwent repeat neuroimaging 4-5 weeks later. Results: Seventy-two participants (14.1 years; $95 \%$ CIs: $13.5,14.8$ ) underwent neuroimaging at 40 days post-injury. Global CBF was significantly higher in the symptomatic group compared to healthy controls, and lower in the asymptomatic group $(F(2,57) 9.734 p<0.001)$. Symptomatic children had increased CBF in the frontal and occipital regions, and asymptomatic children had decreased $\mathrm{CBF}$ in the temporal regions compared to healthy controls. CBF decreased in symptomatic children over time. CBF was a predictor of cognition $(\mathrm{R} 2=0.235 ; \mathrm{p}=0.001)$. Conclusions: Cerebral perfusion is altered in children with $\mathrm{mTBI}$ and is associated with recovery trajectory. Asymptomatic children had decreased CBF suggesting cerebral recovery is ongoing. Further longitudinal studies are required to determine if these perfusion patterns continue to change over time. 